\title{
UNC-13 and UNC-10/Rim Localize Synaptic Vesicles to Specific Membrane Domains
}

\author{
Robby M. Weimer, ${ }^{1 \star}$ Elena 0. Gracheva, ${ }^{2 \star}$ Olivier Meyrignac, ${ }^{1}$ Kenneth G. Miller, ${ }^{3}$ Janet E. Richmond, ${ }^{2}$ and \\ Jean-Louis Bessereau ${ }^{1}$ \\ ${ }^{1}$ Ecole Normale Supérieure, Biologie Cellulaire de la Synapse, and Institut National de la Santé et de la Recherche Médicale, U789, F-75005 Paris, France, \\ ${ }^{2}$ Department of Biological Sciences, University of Illinois-Chicago, Chicago, Illinois 60607, and ${ }^{3}$ Program in Molecular, Cell, and Developmental Biology, \\ Oklahoma Medical Research Foundation, Oklahoma City, Oklahoma 73104
}

Synaptic vesicles undergo a maturation step, termed priming, in which they become competent to fuse with the plasma membrane. To morphologically define the site of vesicle priming and identify fusion-competent synaptic vesicles, we combined a rapid physical-fixation technique with immunogold staining and high-resolution morphometric analysis at Caenorhabditis elegans neuromuscular junctions. In these presynaptic terminals, a subset of synaptic vesicles contact the plasma membrane within $\sim 100$ nm of a presynaptic dense projection. UNC-13, a protein required for vesicle priming, localizes to this same region of the plasma membrane. In an unc-13 null mutant, few synaptic vesicles contact the plasma membrane, suggesting that membrane-contacting synaptic vesicles represent the morphological correlates of primed vesicles. Interestingly, a subpopulation of membrane-contacting vesicles, located within $30 \mathrm{~nm}$ of a dense projection, are unperturbed in unc-13 mutants. We show that UNC-10/Rim, a protein implicated in presynaptic plasticity, localizes to dense projections and that loss of UNC-10/Rim causes an UNC-13-independent reduction in membrane-contacting synaptic vesicles within $30 \mathrm{~nm}$ of the dense projections. Our data together identify a discrete domain for vesicle priming within $100 \mathrm{~nm}$ of dense projections and further suggest that UNC-10/Rim and UNC-13 separately contribute to the membrane localization of synaptic vesicles within this domain.

Key words: C. elegans; neuromuscular junction; exocytosis; postembedding immunogold labeling; high-pressure freezing; electron microscopy

\section{Introduction}

The spatial relationship between sites of synaptic vesicle exocytosis and postsynaptic neurotransmitter receptors is a critical parameter underlying the biophysical properties of synaptic transmission (Cathala et al., 2005). Within a synaptic bouton, vesicle exocytosis occurs at "active zones," identified by electron microscopy as a presynaptic specialization containing electron-dense projections that lie along the presynaptic membrane (Couteaux and Pecot-Dechavassine, 1970; Heuser et al., 1979; Zhai and Bellen, 2004). Although little is known regarding the mechanisms that spatially confine exocytosis, recent work has begun to elucidate the molecular mechanisms driving exocytosis (Sudhof, 2004).

Received April 4, 2006; revised June 14, 2006; accepted June 15, 2006.

This work was supported by National Institutes of Health R01 Grants MH62400 (K.G.M.) and NS41477 (J.E.R.) and by an Avenir grant from the Institut National de la Santé et de la Recherche Médicale (INSERM) (J.-L.B.). R.M.W. was supported by an INSERM fellowship. We thank Philippe Rostaing and Antoine Triller for supportive interactions throughout this project; Ivy Weimer for data analysis; Michael Schade, Angela Thomure (INSERM), Linda Juarez, and Kristina Jarosius (Research Resources Center Electron Microscopy facility, University of Illinois-Chicago, Chicago, IL) and Jay Campbell (Laboratory for Optical and Computational Instrumentation, University of Wisconsin-Madison, Madison, WI) for technical support; and Serge Marty, Brain Ackley, and David Featherstone for critical reading of this manuscript. We also thank Michael Nonet, Mei Zhen, and Alexander van der Bliek for contributing antibodies. *R.M.W. and E.O.G. contributed equally to this work.

Correspondence should be addressed to Dr. Janet E. Richmond, Department of Biological Sciences, University of Illinois-Chicago, 840 West Taylor Street, Chicago, IL 60607. E-mail: jer@uic.edu.

R. M. Weimer's present address: Cold Spring Harbor Laboratory, 1 Bungtown Road, Cold Spring Harbor, NY 11724. DOI:10.1523/JNEUROSCI.2350-06.2006

Copyright $\odot 2006$ Society for Neuroscience $\quad 0270-6474 / 06 / 268040-08 \$ 15.00 / 0$
Synaptic vesicle exocytosis requires members of the soluble $N$-ethylmaleimide-sensitive factor attachment protein (SNAP) receptor (SNARE) protein family: the vesicle protein synaptobrevin and the plasma membrane proteins SNAP-25 and syntaxin (Sollner et al., 1993; Broadie et al., 1995; Hanson et al., 1997). SNAREs interact to form SNARE complexes (Fasshauer, 2003) that "prime" vesicles for exocytosis (Lonart and Sudhof, 2000). However, in solution syntaxin adopts a "closed" configuration in which its $\mathrm{N}$ terminus occludes the syntaxin SNARE domain from SNARE pairing (Dulubova et al., 1999). UNC-13, a presynaptic protein required for priming (Maruyama and Brenner, 1991; Aravamudan et al., 1999; Richmond et al., 1999), interacts with the $\mathrm{N}$ terminus of syntaxin in vitro (Brose et al., 2000; Madison et al., 2005; Stevens et al., 2005) and is thought to promote priming by facilitating the "opening" of syntaxin (Stevens et al., 2005). Consistent with this model, priming is rescued in unc-13 mutants overexpressing an "open" form of syntaxin (Richmond et al., 2001).

The SNARE complex crystal structure predicts that SNARE pairing in trans brings the vesicle into close proximity to the plasma membrane (Sutton et al., 1998); therefore, primed vesicles should contact the plasma membrane. However, previous analysis of synapses in which SNARE proteins (Hunt et al., 1994; Broadie et al., 1995; Deak et al., 2004), UNC-13 (Aravamudan et al., 1999; Augustin et al., 1999; Richmond et al., 1999; Varoqueaux et al., 2002), or UNC-10/Rim, an UNC-13 interacting 
protein (Betz et al., 2001; Koushika et al., 2001; Schoch et al., 2002) were perturbed did not reveal a decrease in membranecontacting vesicles. Perhaps translocation to the plasma membrane occurs before SNARE pairing. Alternatively, typical fixation conditions may not distinguish between membranecontacting primed vesicles and vesicles close to the plasma membrane.

To study the distribution of vesicles at the active zone, we used a fixation technique capable of preserving tissues with minimal structural perturbation: high-pressure freezing (Gilkey and Staehelin, 1986). We show that a subpopulation of synaptic vesicles contacts the plasma membrane within $100 \mathrm{~nm}$ of dense projections and that this region contains UNC-10/Rim and UNC-13. In the absence of UNC-13, the number of vesicles contacting the plasma membrane is greatly reduced, with the exception of those abutting the dense projection. We show that the localization of vesicles adjacent to the dense projection requires UNC-10/Rim, suggesting that UNC-10/Rim targets vesicles to the dense projection in an UNC-13-independent manner. Furthermore, our data imply that molecular events underlying UNC-13-dependent priming transitions vesicles to the plasma membrane before fusion.

\section{Materials and Methods}

High-pressure freezing and freeze substitution. Wild-type (N2 Bristol) (Brenner, 1974), unc-13(s69) (Kohn et al., 2000), unc-10(md1117) (Koushika et al., 2001), and unc-13(s69);unc-10(md1117) (kindly provided by Dr. M. Nonet, Washington University in St. Louis, St. Louis, $\mathrm{MO}$ ) animals were prepared for electron-microscopic analysis as described previously (Weimer, 2006). For morphometric analysis of wildtype and mutant animals, young adult hermaphrodites were vitrified then freeze substituted in $0.1 \%$ tannic acid and $0.5 \%$ gluteraldehyde in acetone for $4 \mathrm{~d}$ at $-90^{\circ} \mathrm{C}$ and in $2 \%$ osmium tetroxide in acetone for $16 \mathrm{~h}$ at $-25^{\circ} \mathrm{C}$ before embedding in Araldite (at $60^{\circ} \mathrm{C}$ for $48 \mathrm{~h}$ ). Serial sections ( $\sim 50 \mathrm{~nm}$ thickness) were collected on formvar carbon-coated copper grids (EMS, FCF2010-Cu). For immunoanalysis, young adult hermaphrodites were vitrified and freeze substituted in either $1.5 \%$ uranyl acetate in methanol at $-90^{\circ} \mathrm{C}$ for $7 \mathrm{~d}$ (Rostaing et al., 2004) or $0.1 \% \mathrm{KMnO}_{4}$ in acetone for $4 \mathrm{~d}$ at $-90^{\circ} \mathrm{C}$ before embedding in Lowicryl $\mathrm{HM} 20$ at $-45^{\circ} \mathrm{C}$ (Weimer, 2006).

Postembedding immunogold labeling. Thin $(\sim 50 \mathrm{~nm})$ sections from Lowicryl-embedded samples were collected as ribbons on formvarcoated nickel slot grids and immunogold labeled in a similar manner to that described previously (Rostaing et al., 2004). Antibody sources and dilutions are as follows: UNC-10 (Koushika et al., 2001), 1:7500; DYN-1 (Labrousse et al., 1998), 1:100; UNC-49 (Gally and Bessereau, 2003), 1:10; and UNC-13 (Charlie et al., 2006), 1:80. UNC-10 and UNC-49 staining was performed on sections from two animal that were fixed separately, UNC-13 staining data were collected from two animals fixed in the same freeze, and DYN-1 data are from one animal. For each antibody, the staining experiment was repeated; each staining experiment consisted of 10 grids with more than five sections per grid. For each primary antibody, the specificity of staining was judged by the signal-tonoise ratio. This was calculated by dividing the gold-particle density within every neuron (DYN-1), within a $30 \mathrm{~nm}$ radius of every dense projection (UNC-10 and UNC-13), or in the muscle within a $100 \mathrm{~nm}$ radius of every GABAergic dense projection (UNC-49) by the goldparticle density within the adjacent cuticle and Escherichia coli on the section. In all cases, the signal-to-noise ration was $>20$.

Electron microscopy. Before examination, some sections were counterstained by incubating with $2.5 \%$ uranyl acetate in $70 \%$ methanol for 4 min, followed, after washing, with Reynold's lead citrate for $2 \mathrm{~min}$. Sections were imaged at $\sim 20,000-100,000 \times$ magnification on either a Philips (Eindhoven, The Netherlands) or JEOL (Peabody, MA) JEM-1220 transmission electron microscope fitted with a Gatan (Pleasanton, CA) $300 \mathrm{~W}$ digital camera.

Data analysis. Measurements were collected from digital micrographs using ImageJ (rsb.info.nih.gov/ij/) and analyzed using Prism (Graph Pad, San Diego, CA). For the morphometric analysis of wild type and mutants, profiles were scored blind.

\section{Results}

The ultrastructure of Caenorhabditis elegans neuromuscular junctions preserved by high-pressure freezing and freeze substitution

C. elegans is a powerful model organism to identify genes involved in synaptic function. However, to fully understand the effect of mutations on the synapse requires high-resolution ultrastructural analysis. We have shown that high-pressure freezing provides a unique opportunity to examine the ultrastructure of synapses in C. elegans (Rostaing et al., 2004; Yeh et al., 2005). In contrast to traditional fixation conditions, an entire living adult hermaphrodite can be physically immobilized in amorphic ice within milliseconds (for discussion, see Weimer, 2006). This rapid physical immobilization minimizes the structural rearrangement that can occur during fixation. To study the detailed ultrastructure of neuromuscular junctions, we immobilized adult $C$. elegans hermaphrodites by high-pressure freezing, embedded frozen animals in resin after a freeze-substitution step, and collected serial sections for observation at the electron microscope.

In cross section, neuronal processes appeared round and contained numerous microtubules after high-pressure freezing immobilization and embedding (Fig. 1A). Presynaptic profiles, dense-core vesicles, and clear-core synaptic vesicles were abundant and easily distinguished. In contrast with traditional fixation conditions used to preserve C. elegans, the presynaptic specialization could be resolved into individual substructures under these fixation conditions (supplemental Fig. 1, available at www. jneurosci.org as supplemental material); specifically, the presynaptic specialization consisted of one to several round electrondense projections (diameter, $\sim 30 \mathrm{~nm}$ ).

To characterize the distribution of cellular structures such as microtubules and synaptic vesicles within a presynaptic profile, we mapped their position relative to two landmarks: we measured the APM (distance to the plasma membrane) and the dDP (distance to the center of the nearest dense projection) for individual structures (Fig. $1 B$ ). Sections that did not contain a discernable dense projection were excluded from our analysis because the resolution in the $z$-axis $(\sim 50 \mathrm{~nm}$, the average section thickness) is much lower than that in the $x-y$ axes. Using this mapping strategy, distinct distribution patterns could be resolved for synaptic vesicles compared with dense-core vesicles and microtubules (Fig. 1C). Synaptic vesicles clustered near dense projections (median dDP, $191 \mathrm{~nm}$ ), whereas dense-core vesicles and microtubules were located distal to synaptic vesicles (median $\mathrm{dDP}, 277$ and $362 \mathrm{~nm}$, respectively).

Within the population of synaptic vesicles sampled $(n=2074$; 91 profiles; seven animals), a subset $(n=226)$ contacted the plasma membrane. We characterized the distribution of these membrane-associated vesicles by measuring the circumferential distance along the plasma membrane between the vesicles and the center of the nearest dense projection (Fig. 1B, dDP-M). More than half of the membrane-associated synaptic vesicles contacted the plasma membrane within a narrow region of a presynaptic profile, (i.e., within $100 \mathrm{~nm}$ of a dense projection; $n=116$ ) (Fig. $1 D)$, which is reflected by the median dDP-M value, $94.2 \mathrm{~nm}$. The remaining membrane-associated vesicles $(n=110)$ were evenly distributed along the membrane at distances $>100 \mathrm{~nm}$ from a dense projection (range, 101-805 nm). 


\section{Proteins required for synaptic vesicle exocytosis and endocytosis localize to distinct regions of the plasma membrane}

Membrane-associated vesicles likely represent intermediates in the trafficking of synaptic vesicles at the plasma membrane. Specifically, they could correspond to synaptic vesicles undergoing exocytosis to release neurotransmitter or endocytosis to recycle vesicle components from the plasma membrane. To identify the location within synaptic terminals where these events occur, we labeled synaptic proteins of known function using postembedding immunogold staining and mapped their distribution relative to the plasma membrane and the nearest dense projection. We have demonstrated previously that SYD-2/liprin, a membrane-associated protein required for the proper organization of the active zone (Zhen and Jin, 1999), is restricted to the presynaptic plasma membrane (Yeh et al., 2005). Analysis of this original data set using the mapping strategy described above revealed that SYD-2 localization is restricted to the plasma membrane at the base of dense projections; the median distance between SYD-2-associated gold particles to the center of the nearest dense projection was $11.5 \mathrm{~nm}$, and the average diameter of a dense projection under these fixation conditions is $29.6 \pm$ $4.8 \mathrm{~nm}$.

UNC-13 and UNC-10/Rim are presynaptic proteins required for synaptic vesicle exocytosis. Antibodies specific for the fulllength C. elegans UNC-13 isoform, UNC13L (Charlie et al., 2006), localized gold particles primarily to the plasma membrane (mean dPM, $16.9 \pm$ $49.3 \mathrm{~nm} ; n=14$ particles; 10 profiles). Nearly all (13 of 14 ) of the gold particles were within $30 \mathrm{~nm}$ of the plasma membrane, therefore considered to be membrane associated. The majority of these particles were localized near dense projections (median dDP-M, $46 \mathrm{~nm}$ ) (Fig. 2A,D). Antibodies directed against C. elegans UNC10/Rim (Koushika et al., 2001) consistently labeled a restricted region of the presynapse. UNC-10/Rim-associated gold particles were localized to the plasma membrane (mean dPM, $33.3 \pm 52.5$ $\mathrm{nm} ; n=25$ particles; 10 profiles). Twenty of these particles were within $30 \mathrm{~nm}$ of the plasma membrane; they were localized just lateral to dense projections (median dDP-M, $26.5 \mathrm{~nm}$ ) (Fig. $2 B, D$ ). These data suggest that the proteins required to prime synaptic vesicles for exocytosis are localized at the plasma membrane within $\sim 100 \mathrm{~nm}$ of a dense projection.

If UNC-13 and Rim define sites of exocytosis, then neurotransmitter receptors should be localized juxtaposed to these sites. To compare the localization of neurotransmitter receptors to dense projections, we stained $\mathrm{GABA}_{\mathrm{A}}$ receptors within bodywall muscles. An antibody directed against UNC-49, the GABA receptor subunit in C. elegans (Bamber et al., 1999; Gally and Bessereau, 2003), localized gold particles to the postsynaptic plasma membrane (mean, $8 \pm 6.9 \mathrm{~nm} ; n=64$ particles; nine

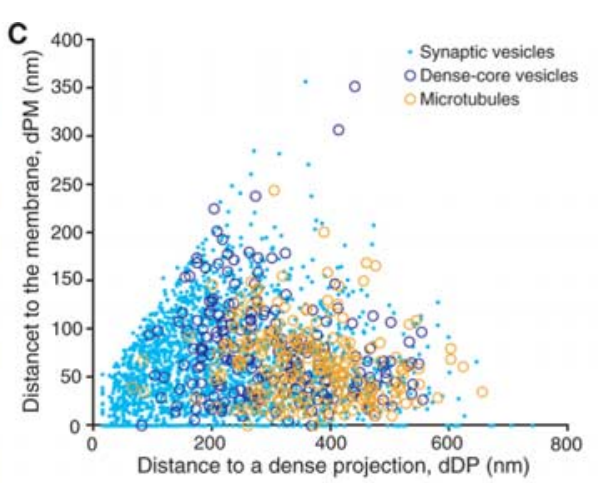

D

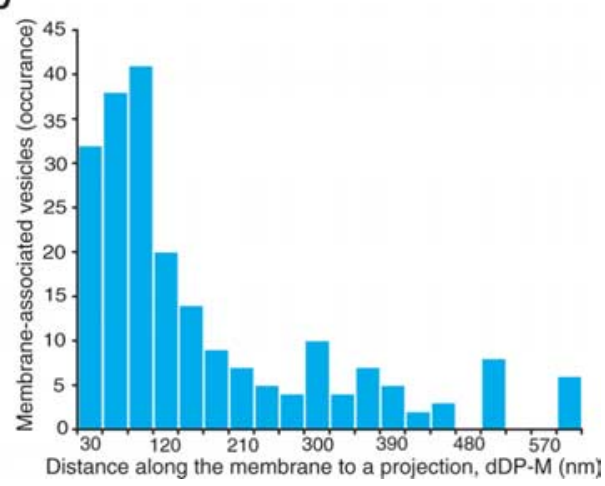

Figure 1. The morphology and mapping of molecular domains at C. elegans neuromuscular junctions after high-pressure freezing and freeze substitution. $A$, Example of a cross section through a C. elegans neuromuscular junction. High-pressure freezing (Dynaptic components at neuromuscular junctions; these include microtubules (MT, orange), dense-core vesicles (DCV pure), synaptic vesicles (SV, blue), and dense projections (DP, black arrow). Scale bar, $100 \mathrm{~nm}$. B, Structures at synapses were bution of plasma membrane-contacting synaptic vesicles relative to the center of the closest dense projection measured as the distance along the plasma membrane (dDP-M). Data are grouped into $30 \mathrm{~nm}$ bins, the average diameter of a synaptic vesicle.

profiles) juxtaposed to presynaptic terminals (Fig. 2E). To correlate the distribution of gold particles relative to dense projections, we measured the distance between UNC-49-associated gold particles and the line perpendicular to the presynaptic plasma membrane below the nearest dense projection (Fig. $1 \mathrm{~B}$, dDP-Post). Gold particles were distributed lateral to dense projections (median dDP-Post, $18.5 \mathrm{~nm}$ ) (Fig. 2E,F). This distribution mirrored that of UNC-13 and UNC-10/Rim (Fig. 2D) and is consistent with the idea that UNC-13 and UNC-10/Rim are localized to sites of neurotransmitter release.

After fusion with the plasma membrane, synaptic vesicles are recycled by endocytosis. This process requires the cytoplasmic GTPase dynamin for the budding of vesicles from the plasma membrane (van der Bliek and Meyerowitz, 1991; Clark et al., 1997). Ultrastructural analysis of endocytosis intermediates in fly mutants or at lamprey synapses suggested that the area of endocytosis does not overlap with exocytosis (Koenig et al., 1989; Shupliakov et al., 1997). Consistent with these morphological observations, antibodies directed against C. elegans dynamin, DYN-1 (Labrousse et al., 1998), localized gold particles to cytoplasmic and plasma membrane regions distant from dense projections (Fig. 2C). The cytoplasmic distribution of DYN-1 at neuromuscular junctions was centered $\sim 323 \pm 134 \mathrm{~nm}(n=57$ 

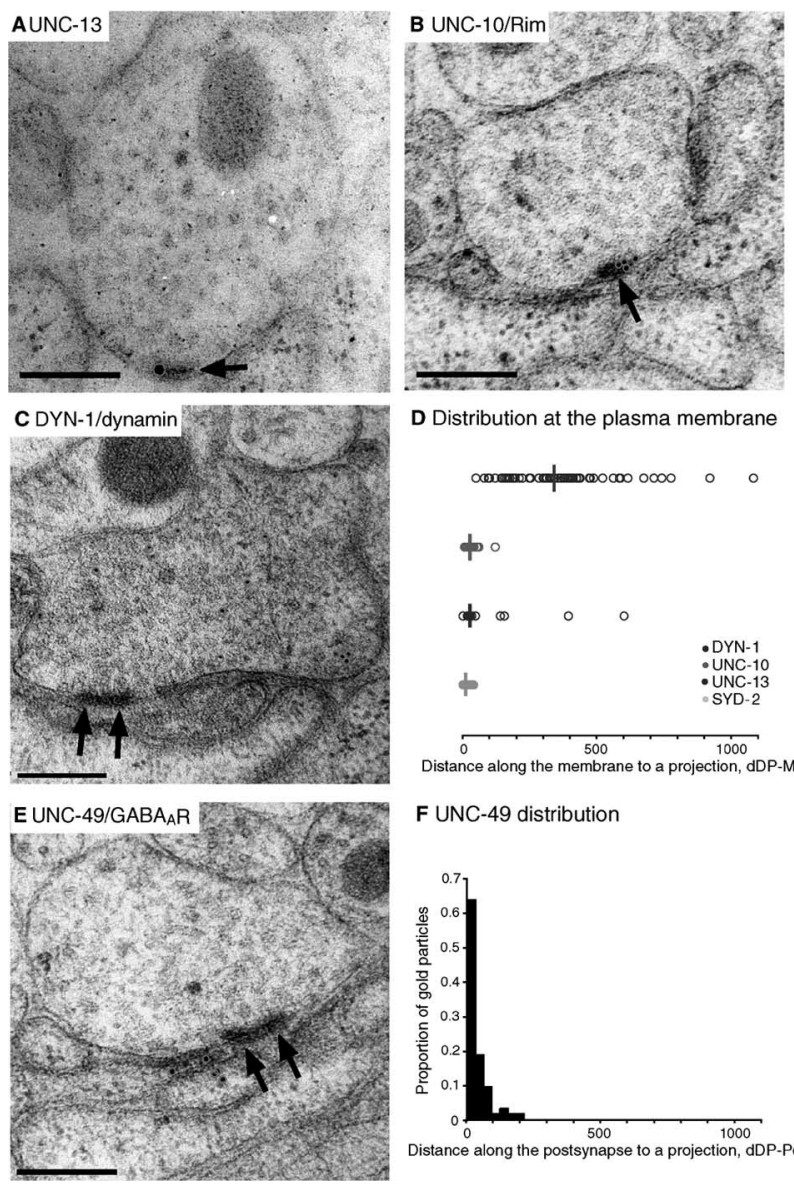

D Distribution at the plasma membrane

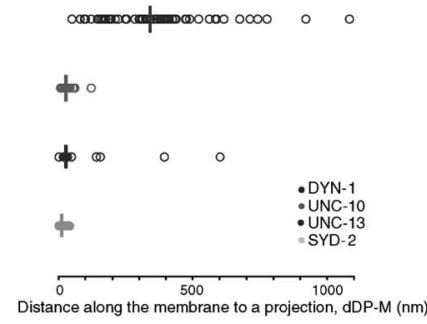

F UNC-49 distribution

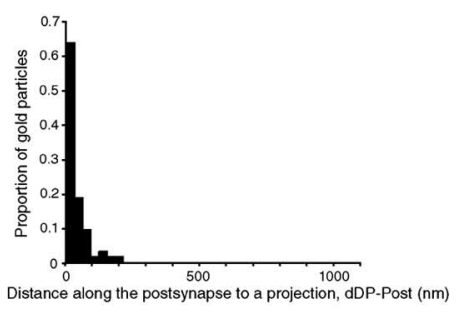

Figure 2. Mapping of molecular domains at the neuromuscular junction using immunogold labeling. Immunogold particles that label specific synaptic proteins were mapped at neuromuscular junctions. $A-C, E$, Representative micrographs of the immunogold labeling (circled in red) of UNC-13 $(\boldsymbol{A})$, UNC-10/Rim $(\boldsymbol{B})$, DYN-1/dynamin $(\boldsymbol{C})$, and UNC-49/GABA receptor $(\boldsymbol{E})$. $\boldsymbol{D}$, The distribution of membrane-associated gold particles relative to the nearest dense projection, measured as the distance along the plasma membrane (dDP-M). Each circle represents an individual measurement. The median value is indicated with a line. The presynaptic proteins UNC-13 and UNC-10/Rim are predominantly localized near dense projections, whereas dynamin is distributed laterally. To illustrate the distribution of a protein intimately associated with dense projections, the previously published SYD-2 immunogold labeling data set (Yeh et al., 2005) was reanalyzed using the outlined mapping strategy and plotted. $\boldsymbol{F}$, Distribution of UNC-49-associated gold particles. Distances were measured from gold particles to the line perpendicular to the plasma membrane below the dense projection (dDP-Post) (see Fig. 1B).

particles; 10 profiles) from the center of the nearest dense projection and likely reflects the location of membrane trafficking pathways (i.e., endosomal, etc.) within a presynaptic bouton. More than one-third of the total gold particles were within $30 \mathrm{~nm}$ of the plasma membrane, thus considered membrane associated. In fact, the majority of these gold particles were associated with clear vesicles contacting the plasma membrane (18 of 30). The plasma membrane-associated DYN-1 gold particles were distributed over a wide range of distances relatively far from a dense projection (median dDP-M, $340.5 \mathrm{~nm}$ ), suggesting that DYN-1mediated endocytosis occurs at regions of the plasma membrane away from dense projections.

Together, these results identify discrete functional domains at presynaptic nerve terminals that have a unique molecular composition. These domains are spatially organized around dense projections, presumably to effectively regulate presynaptic pathways involved in synaptic transmission.

\section{Synaptic vesicle priming transitions vesicles to the} plasma membrane

Our immunogold labeling experiments indicate that the vesicle priming factor, UNC-13, is localized to the membrane near dense projections. This staining pattern colocalizes with the majority of membrane-associated synaptic vesicles within a presynaptic profile (Fig. $1 D$ ). Therefore, we hypothesized that a fraction of these membrane-associated vesicles likely represent fusion competent, or primed, synaptic vesicle. Hence, mutations that disrupt UNC-13 function might specifically alter the distribution of these membrane-associated synaptic vesicles.

To test this prediction, we compared the distribution of synaptic vesicles in the severe loss-of-function $u n c-13$ mutant $u n c$ 13(s69) (Kohn et al., 2000), with that in the wild type preserved by high-pressure freezing and freeze substitution (Fig. 3). Compared with wild type, neuromuscular junctions in unc-13 mutants contained significantly more synaptic vesicles (wild type: $22.2 \pm 0.5$ synaptic vesicles per profile, $n=70$ profiles, four animals; unc-13(s69): $25.2 \pm 0.8$ synaptic vesicles per profile, $n=$ 50 profiles, four animals; $p=0.001$ ) (Fig. $3 A$ ). The accumulation of vesicles in unc-13(s69) by high-pressure freezing was consistent with, although less pronounced, than previous ultrastructural studies of $u n c-13$ mutants using conventional fixation (Aravamudan et al., 1999; Augustin et al., 1999; Richmond et al., 1999; Varoqueaux et al., 2002). However, in contrast to previous studies, we found that fewer synaptic vesicles per synaptic profile contact the plasma membrane in unc-13 mutants (wild type: $9.8 \pm 0.4 \%$ membrane-contacting synaptic vesicles per profile; unc-13(s69): $4.1 \pm 0.4 \%$ membrane-contacting synaptic vesicles per profile; $p<0.0001)$. The discrepancy between these results and previous published studies likely stems from differences in fixation conditions: high-pressure freezing is capable of near instantaneous fixation, whereas traditional chemical fixation occurs over time scales of minutes. The redistribution of synaptic vesicle populations associated with chemical fixation have been noted in previous comparisons between chemical and rapid freeze fixation (Rizzoli and Betz, 2004). Overall, we observed fewer synaptic vesicles along the entire plasma membrane in unc-13 mutants (Fig. 3B), with the exception of vesicles immediately adjacent to the dense projection where the fraction of membrane-contacting vesicles was not significantly reduced relative to the wild type ( $p>0.3$ for $30 \mathrm{~nm}$ dDP-M). This overall decrease in membrane-contacting synaptic vesicles in unc-13 mutants is consistent with the hypothesis that UNC-13dependent priming transitions synaptic vesicles to the plasma membrane before exocytosis.

\section{UNC-10/Rim localizes primed vesicles to dense projections}

In the absence of UNC-13, only synaptic vesicles abutting a dense projection contact the plasma membrane, suggesting that an additional pathway, may mediate membrane contact next to dense projections. Because UNC-10/Rim is required for normal levels of vesicle priming (Koushika et al., 2001; Schoch et al., 2002), localizes to dense projections, and interacts with vesicleassociated Rab3a (Wang et al., 1997), we hypothesized that UNC10/Rim mediates membrane contact at dense projections independent of UNC-13. To test this possibility, we compared the distribution of synaptic vesicles between wild type and the unc-10 null mutant unc-10(md1117) (Koushika et al., 2001).

unc-10 mutant synapses contained significantly more synaptic vesicles than wild type $(25.7 \pm 0.7$ synaptic vesicles per profile, $n=59$ profiles, four animals; $p<0.0001$ relative to the wild type) (Fig. 3A), consistent with a defect in exocytosis. Overall, the pro- 
A

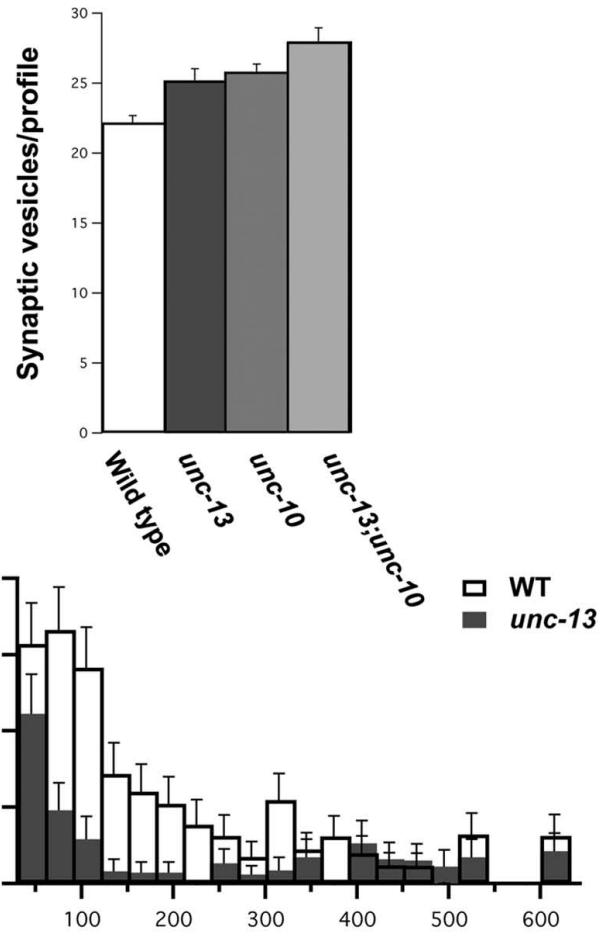

C

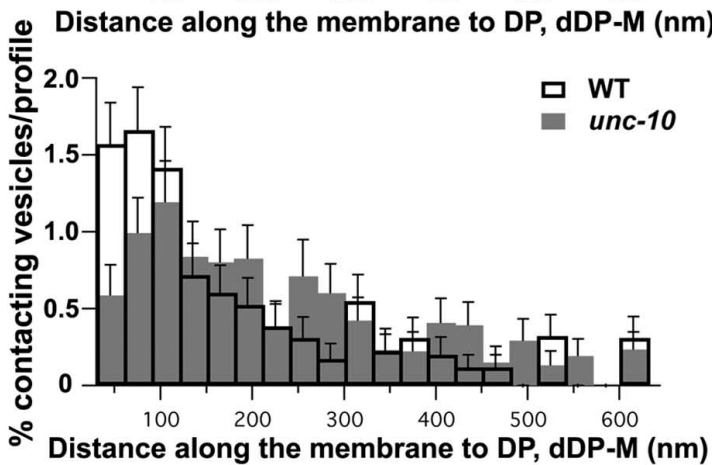

D

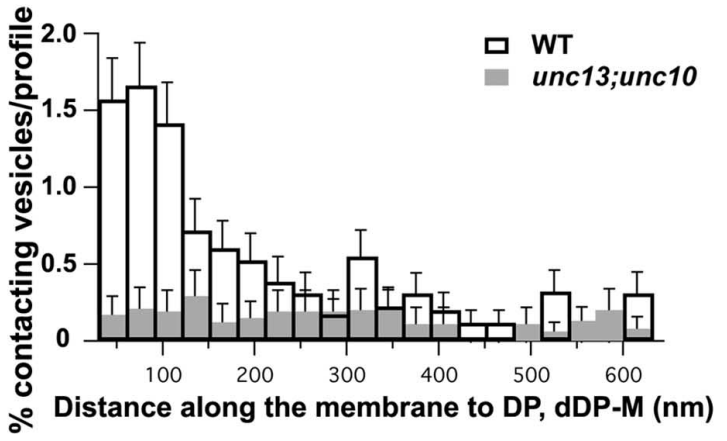

Figure 3. Morphological analysis of unc-13 and unc-10 mutant synapses. A, Quantification of the total number of synaptic vesicles per presynaptic profile for wild-type, unc-13, unc-10, and unc-13(s69); unc-10(md117) synapses. The total number of synaptic vesicles per profile was significantly elevated in unc-13(s69), unc-10(md1117), and unc-13(s69); unc-10(md117) mutants. $\boldsymbol{B}$, Distribution along the plasma membrane of membrane-contacting synaptic vesicles with respect to the dense projection in unc-13(569) and wild-type animals. There is a dramatic reduction in the ratio of plasma membrane-contacting synaptic vesicle $>30 \mathrm{~nm}$ away from a dense projection in unc-13(569). C, The distribution of plasma membrane-contacting vesicles with respect to dense projections in unc-10(md1117) and wild-type animals. There is a decrease in the ratio of synaptic vesicles contacting the plasma membrane immediately adjacent to dense projections in unc-10(md1117) mutants. $\boldsymbol{D}$, Distribution of plasma membranecontacting synaptic vesicles with respect to the dense projection in unc-13(569); unc10(md1117) and wild-type animals. There is a dramatic reduction in the ratio of plasma membrane-contacting synaptic vesicles throughout the distribution, including the bins adjacent to the dense projection. All data are plotted as mean $\pm \mathrm{SEM}$; data in $\boldsymbol{B}-\boldsymbol{D}$ are grouped into $30 \mathrm{~nm}$ bins, which is the average diameter of a synaptic vesicle. WT, Wild type. portion of membrane-contacting synaptic vesicles was normal in unc-10 mutants (wild type, $9.8 \pm 0.4 \%$ membrane-contacting synaptic vesicles per profile; unc-10(md1117), $10.2 \pm 0.35 \%$ membrane-contacting synaptic vesicles per profile; $p>0.5$ ); however, there was a specific and significant decrease in the proportion of vesicles contacting the membrane adjacent to a dense projection relative to the wild type ( $p=0.026$ for $30 \mathrm{~nm} \mathrm{dDP-M}$ ) (Fig. 3C). This suggests that UNC-10/Rim is required for targeting membrane-contacting synaptic vesicles to dense projections and may account for the remaining pool of vesicles that contact the plasma membrane near dense projections in unc-13 mutants.

To directly test whether the residual contacting vesicles observed in unc-13(s69) require UNC-10, we analyzed unc-13; unc-10 double mutants. Phenotypically unc-13(s69);unc10(md1117) mutants appear as paralyzed as unc-13(s69) mutants. Consistent with each of the single mutants, synapses from the double mutant contained more synaptic vesicles than the wild type $(28 \pm 1.0$ synaptic vesicles per profile, $n=37$ profiles, two animals; $p<0.0001$ relative to the wild type) (Fig. $3 A)$. However, few synaptic vesicles contact the plasma membrane (Fig. $3 D$ ). The proportion of membrane-contacting synaptic vesicles in the double mutant was more profoundly reduced than unc-13 alone [2.9 $\pm 0.4 \%$ membrane-contacting synaptic vesicles per profile; $p=0.03$ relative to $u n c-13(s 69)]$. Furthermore, the proportion of synaptic vesicles that contact the membrane next to a dense projection was significantly reduced in the double mutant compared with unc-13(s69) mutants ( $p=0.0047$ for $30 \mathrm{~nm}$ dDP-M) (Fig. $3 B, D$ ) These data suggest that UNC-10 plays a role in recruiting synaptic vesicles to the membrane adjacent to the dense projection in an UNC-13-independent process.

\section{Discussion}

Using high-pressure freezing/freeze substitution, we preserved $C$. elegans synapses for high-resolution immunocytochemical and morphometric analysis. Our data define discrete functional domains within synapses involved in vesicle exocytosis and endocytosis. Specifically, our results indicate that the exocytotic proteins UNC-13 and UNC-10/Rim localize near presynaptic dense projections, whereas dynamin, required for vesicle recycling, associates with the plasma membrane in the periactive zone. Morphometric analysis of unc-13 mutants indicate that UNC-13dependent priming transitions vesicles to the plasma membrane proximal to the dense projection before fusion, whereas UNC10/Rim targets vesicles to the dense projection in an UNC-13independent manner.

\section{Ultrastructural preservation by high-pressure freezing C. elegans}

The rapid fixation afforded by high-pressure freezing provides an unique opportunity to examine cellular structures in C. elegans. An entire animal can be immobilized in amorphic ice nearly instantaneously, thus its ultrastructure can be examined with minimal structural perturbation. At neuromuscular junctions, the ability of this fixation technique to preserve labile structures is apparent from the vast microtubule network observed in neuronal profiles: microtubules often depolymerize during traditional chemical fixation (Kiss et al., 1990; McMenamin et al., 2003; Rostaing et al., 2004).

The ability to instantly immobilize synaptic vesicles is important for the morphometric analysis of synaptic vesicle populations. Specifically, the time required to preserve C. elegans synapses with traditional protocols is not known. Because animals move for tens of seconds after immersion in a cold hypertonic 
fixative, synaptic vesicles may be redistributed before fixation. Consistent with this notion, the redistribution of synaptic vesicles during chemical fixation has been reported in a previous study that used both chemical and rapid-freeze fixation techniques (Rizzoli and Betz, 2004).

Previous analysis of unc-13 mutants reported a 50\% increase in the total number of synaptic vesicles in presynaptic boutons (Richmond et al., 1999). High-pressure freezing of these mutants found a $<20 \%$ increase in synaptic vesicle number in unc-13 mutants. This result supports the idea that slow chemical fixation might result in a significant redistribution of synaptic vesicles. Moreover, there is no change in the number of synaptic vesicles contacting the plasma membrane in unc-13 (Richmond et al., 1999) or unc-10 (Koushika et al., 2001) mutants when preserved by chemical fixation. Instant vitrification of animals enables the resolution of protein domains and synaptic vesicle populations at neuromuscular junctions based on nanometer differences in distances relative to the plasma membrane and dense projections.

\section{Protein domains and synaptic structures}

At chemical synapses, synaptic transmission is mediated by regulated fusion of synaptic vesicles with the plasma membrane and subsequent recycling. Synaptic vesicle exocytosis and endocytosis use distinct molecular machinery. Endocytosis requires clathrin, clathrin adaptor proteins, and dynamin (Morgan et al., 2002). We found that endogenous dynamin localizes to the plasma membrane away $(>100 \mathrm{~nm}$ ) from dense projections, suggesting that synaptic vesicle endocytosis occurs within this region. Consistent with recent results obtained at lamprey synapses (Evergren et al., 2006), these data represent the first ultrastructural descriptions of dynamin localization in periactive zones. That we found a small subset of membrane-contacting synaptic vesicles in this region of the plasma membrane is also consistent with previous studies of endocytic intermediates at other presynaptic nerve terminals (Koenig et al., 1989; Shupliakov et al., 1997; Harris et al., 2000; Bloom et al., 2003; Rizzoli and Betz, 2004).

Exocytosis requires members of the SNARE protein family (Sollner et al., 1993; Broadie et al., 1995; Hanson et al., 1997) and UNC-13 (Aravamudan et al., 1999; Augustin et al., 1999; Richmond et al., 1999; Varoqueaux et al., 2002) and is regulated by UNC-10/Rim (Koushika et al., 2001; Schoch et al., 2002). The plasma membrane SNARE syntaxin is absolutely required for membrane fusion (Broadie et al., 1995) and is distributed throughout the neuronal plasma membrane (Weimer et al., 2003). However, our data suggest that UNC-13 and UNC-10/ Rim are restricted to sites within $\sim 100 \mathrm{~nm}$ of dense projections. This region of the plasma membrane therefore likely represents sites of synaptic vesicle exocytosis. Consistent with this hypothesis, $\mathrm{GABA}_{\mathrm{A}}$ receptors are localized opposite these putative release sites at GABAergic neuromuscular junctions. The distribution of syntaxin throughout the plasma membrane may reflect additional roles for syntaxin in other membrane trafficking pathways (Foletti et al., 2000).

Our morphological and molecular data support the model of compartmentalized synaptic vesicle cycling at presynaptic terminals, consistent with previous ultrastructural studies. Synaptic vesicle exocytosis occurs at defined sites near dense projections, and recycling of synaptic vesicle components occurs distally. This model implies that synaptic vesicle proteins in the plasma membrane are sorted and transitioned to lateral domains to be recycled.

\section{Priming of synaptic vesicles}

The priming of synaptic vesicles is thought to involve the pairing of the vesicle SNARE synaptobrevin with the plasma membrane SNAREs syntaxin and SNAP-25 (Lonart and Sudhof, 2000) and requires UNC-13 (Aravamudan et al., 1999; Augustin et al., 1999; Richmond et al., 1999; Varoqueaux et al., 2002). The crystal structure of the core SNARE complex predicts that SNARE pairing brings the vesicle membrane into close proximity to the plasma membrane (Sutton et al., 1998). However, previous ultrastructural analysis of SNARE protein (Hunt et al., 1994; Broadie et al., 1995; Deak et al., 2004) or UNC-13 (Aravamudan et al., 1999; Augustin et al., 1999; Richmond et al., 1999; Varoqueaux et al., 2002) perturbations have failed to resolve changes in plasma membrane-contacting synaptic vesicles. This is with the exception of Munc13 1/2 knock-outs in which a significant decrease in contacting vesicle density was observed, which was attributed to an increased active zone area (Varoqueaux et al., 2002). Here, we report that unc-13 mutants in C. elegans do, in fact, exhibit a significant decrease in the population of plasma membranecontacting vesicles near dense projections. Our ability to detect this defect likely stems from our fixation technique and adds credence to the hypothesis that UNC-13-dependent priming, through SNARE protein interactions, transitions synaptic vesicles to the plasma membrane before fusion. In addition, our data suggest that at $C$. elegans neuromuscular junctions, primed vesicles can be identified and studied based on the following morphological criteria: distance to the plasma membrane and the dense projection. The use of high-pressure freezing in other organisms might extend this observation to other types of synapses.

\section{The pathways of vesicle priming}

Our ultrastructural analysis of $u n c-13$ mutants suggests that vesicle priming results in the transition of synaptic vesicles to the plasma membrane. However, we found significant differences between the ultrastructural phenotype of unc-13 mutants and that of unc-10, a gene encoding UNC-10/Rim, implicated in synaptic plasticity and regulation of priming. Specifically, unc-13 mutant synapses contain few membrane-associated vesicles between 60 and $120 \mathrm{~nm}$ from a dense projection, with less change in the population within $30 \mathrm{~nm}$, whereas unc-10 mutants contain few vesicles within $30 \mathrm{~nm}$ of a dense projection but have relatively normal levels between 60 and $120 \mathrm{~nm}$. This difference in vesicle distribution likely reflects the different roles of each protein.

A current model for UNC-13-mediated priming suggests that UNC-13 facilitates a conformational switch in syntaxin, enabling syntaxin to form SNARE complexes (Brose et al., 2000), either through a direct interaction with syntaxin (Madison et al., 2005; Stevens et al., 2005) or through an indirect pathway (Basu et al., 2005). UNC-10/Rim, on the other hand, is not absolutely required for vesicle priming, a significant proportion of vesicles remaining fusion competent in the absence of UNC-10/Rim (Koushika et al., 2001; Schoch et al., 2002). UNC-10/Rim interacts directly with UNC-13 but not syntaxin, and this UNC-10/ Rim-UNC-13 interaction is required for UNC-10/Rim function (Betz et al., 2001). Recent evidence indicates that Rim recruits Munc13 to the synapse and is required to stabilize Munc13 protein levels (Andrews-Zwilling et al., 2006). Through this interaction with UNC-13, UNC-10/Rim is likely to regulate synaptic vesicle priming, although in C. elegans, UNC-13 is still detected at the synapse of unc-10 mutants (Koushika et al., 2001).

Our finding that UNC-10/Rim localization is restricted to dense projections and that this region contains fewer membranecontacting vesicles in $u n c-10$ mutants suggests that UNC-10/Rim 
functions at a spatially restricted region adjacent to dense projections to localize vesicles. This hypothesis is also supported by the demonstrated protein-protein interaction between UNC-10/ Rim and $\alpha$-liprin (Ko et al., 2003), which is localized at dense projections (Yeh et al., 2005). UNC-10/Rim also interacts with the synaptic vesicle-associated GTPase Rab3 (Wang et al., 1997) implicated in regulating the release probability of primed synaptic vesicles (Schluter et al., 2004). Mammalian Rab3, Rim, and Munc13 interact to form a tripartite complex (Dulubova et al., 2005). Therefore, the localized distribution of UNC-10/Rim might recruit vesicles to the plasma membrane through its interaction with Rab3 and facilitates subsequent interactions with UNC-13. This Rab3-UNC-10/Rim-UNC-13 interaction also provides a means to localize primed synaptic vesicles in close vicinity to dense projections. Consistent with this model, fewer synaptic vesicles localize near dense projections in C. elegans rab-3 mutants (Nonet et al., 1997). If calcium entry occurs preferentially at this site, regulating the distribution of "primed" synaptic vesicles would account for the "superpriming" role attributed to Rab3a, by maintaining a primed vesicle pool at dense projections via UNC-10-UNC-13 interactions (Schluter et al., 2006).

\section{References}

Andrews-Zwilling YS, Kawabe H, Reim K, Varoqueaux F, Brose N (2006) Binding to RAB3a-interacting-molecule rim regulates the presynaptic recruitment of MUNC13-1 and ubMUNC13-2. J Biol Chem, in press.

Aravamudan B, Fergestad T, Davis WS, Rodesch CK, Broadie K (1999) Drosophila UNC-13 is essential for synaptic transmission. Nat Neurosci 2:965-971.

Augustin I, Rosenmund C, Sudhof TC, Brose N (1999) Munc13-1 is essential for fusion competence of glutamatergic synaptic vesicles. Nature 400:457-461.

Bamber BA, Beg AA, Twyman RE, Jorgensen EM (1999) The Caenorhabditis elegans unc-49 locus encodes multiple subunits of a heteromultimeric GABA receptor. J Neurosci 19:5348-5359.

Basu J, Shen N, Dulubova I, Lu J, Guan R, Guryev O, Grishin NV, Rosenmund C, Rizo J (2005) A minimal domain responsible for Munc13 activity. Nat Struct Mol Biol 12:1017-1018.

Betz A, Thakur P, Junge HJ, Ashery U, Rhee JS, Scheuss V, Rosenmund C, Rettig J, Brose N (2001) Functional interaction of the active zone proteins Munc13-1 and RIM1 in synaptic vesicle priming. Neuron 30:183-196.

Bloom O, Evergren E, Tomilin N, Kjaerulff O, Low P, Brodin L, Pieribone VA, Greengard P, Shupliakov O (2003) Colocalization of synapsin and actin during synaptic vesicle recycling. J Cell Biol 161:737-747.

Brenner S (1974) The genetics of Caenorhabditis elegans. Genetics 77:71-94.

Broadie K, Prokop A, Bellen HJ, O’Kane CJ, Schulze KL, Sweeney ST (1995) Syntaxin and synaptobrevin function downstream of vesicle docking in Drosophila. Neuron 15:663-673.

Brose N, Rosenmund C, Rettig J (2000) Regulation of transmitter release by Unc-13 and its homologues. Curr Opin Neurobiol 10:303-311.

Cathala L, Holderith NB, Nusser Z, DiGregorio DA, Cull-Candy SG (2005) Changes in synaptic structure underlie the developmental speeding of AMPA receptor-mediated EPSCs. Nat Neurosci 8:1310-1318.

Charlie NK, Thomure AM, Schade MA, Miller KG (2006) The Dunce cAMP phosphodiesterase PDE-4 negatively regulates G\{alpha\}s-dependent and G\{alpha\}s-independent cAMP pools in the Caenorhabditis elegans synaptic signaling network. Genetics 173:111-130.

Clark SG, Shurland DL, Meyerowitz EM, Bargmann CI, van der Bliek AM (1997) A dynamin GTPase mutation causes a rapid and reversible temperature- inducible locomotion defect in C. elegans. Proc Natl Acad Sci USA 94:10438-10443.

Couteaux R, Pecot-Dechavassine M (1970) Synaptic vesicles and pouches at the level of "active zones" of the neuromuscular junction. C R Acad Sci Hebd Seances Acad Sci D 271:2346-2349.

Deak F, Schoch S, Liu X, Sudhof TC, Kavalali ET (2004) Synaptobrevin is essential for fast synaptic-vesicle endocytosis. Nat Cell Biol 6:1102-1108.

Dulubova I, Sugita S, Hill S, Hosaka M, Fernandez I, Sudhof TC, Rizo J
(1999) A conformational switch in syntaxin during exocytosis: role of munc18. EMBO J 18:4372-4382.

Dulubova I, Lou X, Lu J, Huryeva I, Alam A, Schneggenburger R, Sudhof TC, Rizo J (2005) A Munc13/RIM/Rab3 tripartite complex: from priming to plasticity? EMBO J 24:2839-2850.

Evergren E, Zotova E, Brodin L, Shupliakov O (2006) Differential efficiency of the endocytotic machinery in tonic and phasic synapses. Neuroscience 141:123-131.

Fasshauer D (2003) Structural insights into the SNARE mechanism. Biochim Biophys Acta 1641:87-97.

Foletti DL, Lin R, Finley MA, Scheller RH (2000) Phosphorylated syntaxin 1 is localized to discrete domains along a subset of axons. J Neurosci 20:4535-4544.

Gally C, Bessereau JL (2003) GABA is dispensable for the formation of junctional GABA receptor clusters in Caenorhabditis elegans. J Neurosci 23:2591-2599.

Gilkey JC, Staehelin LA (1986) Advances in ultrarapid freezing for the preservation of cellular ultrastructure. J Electron Micros Tech 3:177-210.

Hanson PI, Roth R, Morisaki H, Jahn R, Heuser JE (1997) Structure and conformational changes in NSF and its membrane receptor complexes visualized by quick-freeze/deep-etch electron microscopy. Cell 90:523-535.

Harris TW, Hartwieg E, Horvitz HR, Jorgensen EM (2000) Mutations in synaptojanin disrupt synaptic vesicle recycling. J Cell Biol 150:589-600.

Heuser JE, Reese TS, Dennis MJ, Jan Y, Jan L, Evans L (1979) Synaptic vesicle exocytosis captured by quick freezing and correlated with quantal transmitter release. J Cell Biol 81:275-300.

Hunt JM, Bommert K, Charlton MP, Kistner A, Habermann E, Augustine GJ, Betz H (1994) A post-docking role for synaptobrevin in synaptic vesicle fusion. Neuron 12:1269-1279.

Kiss JZ, Giddings TH Jr, Staehelin LA, Sack FD (1990) Comparison of the ultrastructure of conventionally fixed and high pressure frozen/freeze substituted root tips of Nicotiana and Arabidopsis. Protoplasma 157:64-74.

Ko J, Na M, Kim S, Lee JR, Kim E (2003) Interaction of the ERC family of RIM-binding proteins with the liprin-alpha family of multidomain proteins. J Biol Chem 278:42377-42385.

Koenig JH, Kosaka T, Ikeda K (1989) The relationship between the number of synaptic vesicles and the amount of transmitter released. J Neurosci 9:1937-1942.

Kohn RE, Duerr JS, McManus JR, Duke A, Rakow TL, Maruyama H, Moulder G, Maruyama IN, Barstead RJ, Rand JB (2000) Expression of multiple UNC-13 proteins in the C. elegans nervous system. Mol Biol Cell 11:3441-3452.

Koushika SP, Richmond JE, Hadwiger G, Weimer RM, Jorgensen EM, Nonet ML (2001) A post-docking role for active zone protein Rim. Nat Neurosci 4:997-1005.

Labrousse AM, Shurland DL, van der Bliek AM (1998) Contribution of the GTPase domain to the subcellular localization of dynamin in the nematode Caenorhabditis elegans. Mol Biol Cell 9:3227-3239.

Lonart G, Sudhof TC (2000) Assembly of SNARE core complexes prior to neurotransmitter release sets the readily releasable pool of synaptic vesicles. J Biol Chem 275:27703-27707.

Madison JM, Nurrish S, Kaplan JM (2005) UNC-13 interaction with syntaxin is required for synaptic transmission. Curr Biol 15:2236-2242.

Maruyama IN, Brenner S (1991) A phorbol ester/diacylglycerol-binding protein encoded by the unc-13 gene of Caenorhabditis elegans. Proc Natl Acad Sci USA 88:5729-5733.

McMenamin S, Reinsch S, Conway G (2003) Direct comparison of common fixation methods for preservation of microtubules in zebrafish embryos. Biotechniques 34:468-470:472.

Morgan JR, Augustine GJ, Lafer EM (2002) Synaptic vesicle endocytosis: the races, places, and molecular faces. Neuromol Med 2:101-114.

Nonet ML, Staunton JE, Kilgard MP, Fergestad T, Hartwieg E, Horvitz HR, Jorgensen EM, Meyer BJ (1997) Caenorhabditis elegans rab-3 mutant synapses exhibit impaired function and are partially depleted of vesicles. J Neurosci 17:8061-8073.

Richmond JE, Davis WS, Jorgensen EM (1999) UNC-13 is required for synaptic vesicle fusion in C. elegans. Nat Neurosci 2:959-964.

Richmond JE, Weimer RM, Jorgensen EM (2001) An open form of syntaxin bypasses the requirement for UNC-13 in vesicle priming. Nature 412:338-341. 
Rizzoli SO, Betz WJ (2004) The structural organization of the readily releasable pool of synaptic vesicles. Science 303:2037-2039.

Rostaing P, Weimer RM, Jorgensen EM, Triller A, Bessereau JL (2004) Preservation of immunoreactivity and fine structure of adult $C$. elegans tissues using high-pressure freezing. J Histochem Cytochem 52:1-12.

Schluter OM, Schmitz F, Jahn R, Rosenmund C, Sudhof TC (2004) A complete genetic analysis of neuronal Rab3 function. J Neurosci 24:6629-6637.

Schluter OM, Basu J, Sudhof TC, Rosenmund C (2006) Rab3 superprimes synaptic vesicles for release: implications for short-term synaptic plasticity. J Neurosci 26:1239-1246.

Schoch S, Castillo PE, Jo T, Mukherjee K, Geppert M, Wang Y, Schmitz F, Malenka RC, Sudhof TC (2002) RIMlalpha forms a protein scaffold for regulating neurotransmitter release at the active zone. Nature 415:321-326.

Shupliakov O, Low P, Grabs D, Gad H, Chen H, David C, Takei K, De Camilli P, Brodin L (1997) Synaptic vesicle endocytosis impaired by disruption of dynamin-SH3 domain interactions. Science 276:259-263.

Sollner T, Whiteheart SW, Brunner M, Erdjument-Bromage H, Geromanos S, Tempst P, Rothman JE (1993) SNAP receptors implicated in vesicle targeting and fusion. Nature 362:318-324.

Stevens DR, Wu ZX, Matti U, Junge HJ, Schirra C, Becherer U, Wojcik SM, Brose N, Rettig J (2005) Identification of the minimal protein domain required for priming activity of Munc13-1. Curr Biol 15:2243-2248.

Sudhof TC (2004) The synaptic vesicle cycle. Annu Rev Neurosci 27:509-547.

Sutton RB, Fasshauer D, Jahn R, Brunger AT (1998) Crystal structure of a
SNARE complex involved in synaptic exocytosis at 2.4 A resolution. Nature 395:347-353.

van der Bliek AM, Meyerowitz EM (1991) Dynamin-like protein encoded by the Drosophila shibire gene associated with vesicular traffic. Nature 351:411-414

Varoqueaux F, Sigler A, Rhee JS, Brose N, Enk C, Reim K, Rosenmund C (2002) Total arrest of spontaneous and evoked synaptic transmission but normal synaptogenesis in the absence of Munc13-mediated vesicle priming. Proc Natl Acad Sci USA 99:9037-9042.

Wang Y, Okamoto M, Schmitz F, Hofmann K, Sudhof TC (1997) Rim is a putative Rab3 effector in regulating synaptic-vesicle fusion. Nature 388:593-598.

Weimer RM (2006) Preservation of C. elegans tissue via high-pressure freezing and freeze-substitution for ultrastructural analysis and immunocytochemistry. In: C. elegans: methods and applications (Strange K, ed). Totowa, NJ: Humana, in press.

Weimer RM, Richmond JE, Davis WS, Hadwiger G, Nonet ML, Jorgensen EM (2003) Defects in synaptic vesicle docking in unc-18 mutants. Nat Neurosci 6:1023-1030.

Yeh E, Kawano T, Weimer RM, Bessereau JL, Zhen M (2005) Identification of genes involved in synaptogenesis using a fluorescent active zone marker in Caenorhabditis elegans. J Neurosci 25:3833-3841.

Zhai RG, Bellen HJ (2004) The architecture of the active zone in the presynaptic nerve terminal. Physiology (Bethesda) 19:262-270.

Zhen M, Jin Y (1999) The liprin protein SYD-2 regulates the differentiation of presynaptic termini in C. elegans. Nature 401:371-375. 